\title{
OSERF2 controls rice root growth and hormone responses through tuning expression of key genes involved in hormone signaling and sucrose metabolism
}

\author{
Guiqing Xiao ${ }^{1,2} \cdot$ Hua Qin $^{2} \cdot$ Jiahao Zhou $^{2} \cdot$ Ruidang Quan ${ }^{2} \cdot$ Xiangyang Lu ${ }^{1} \cdot$ \\ Rongfeng Huang ${ }^{2} \cdot$ Haiwen Zhang $^{2}$
}

Received: 2 September 2015 / Accepted: 30 November 2015/Published online: 10 December 2015

(C) The Author(s) 2015. This article is published with open access at Springerlink.com

\begin{abstract}
Root determines plant distribution, development progresses, stress response, as well as crop qualities and yields, which is under the tight control of genetic programs and environmental stimuli. Ethylene responsive factor proteins (ERFs) play important roles in plant growth and development. Here, the regulatory function of $O S E R F 2$ involved in root growth was investigated using the gainfunction mutant of OsERF2 (nsf2857) and the artificial microRNA-mediated silenced lines of OsERF2 (AmiOsERF2). nsf2857 showed short primary roots compared with the wild type (WT), while the primary roots of AmiOSERF2 lines were longer than those of WT. Consistent with this phenotype, several auxin/cytokinin responsive genes involved in root growth were downregulated in $n s f 2857$, but upregulated in Ami-OsERF2. Then, we found that $n s f 2857$ seedlings exhibited decreased ABA accumulation and sensitivity to $\mathrm{ABA}$ and reduced ethylene-
\end{abstract}

Guiqing Xiao and Hua Qin have contributed equally to this work.

Electronic supplementary material The online version of this article (doi:10.1007/s11103-015-0416-9) contains supplementary material, which is available to authorized users.

\footnotetext{
Xiangyang Lu

xiangyangcn@163.com

$\triangle$ Rongfeng Huang

rfhuang@caas.cn

$\triangle$ Haiwen Zhang

zhanghaiwen@caas.cn

1 College of Bioscience and Biotechnology, Hunan Agricultural University, Changsha 410128, People's Republic of China

2 Biotechnology Research Institute, Chinese Academy of Agricultural Sciences, Beijing 100081, People's Republic of China
}

mediated root inhibition, while those were the opposite in Ami-ERF2 plants. Moreover, several key genes involved in ABA synthesis were downregulated in nsf 2857 , but unregulated in Ami-ERF2 lines. In addition, OsERF2 affected the accumulation of sucrose and UDPG by mediating expression of key genes involved in sucrose metabolism. These results indicate that OSERF2 is required for the control of root architecture and ABA- and ethyleneresponse by tuning expression of series genes involved in sugar metabolism and hormone signaling pathways.

Keywords OsERF2 - Rice - Root growth - ABA · Ethylene $\cdot$ Sugar

\section{Introduction}

Root systems are central to the acquisition of water and nutrients, plant anchorage, seedling vigor, and responses to various stresses, which have pivotal effects on crop distribution, maximal productivity and yield stability, particularly in arid regions. Root architecture is under the tight control of genetic programs and environmental stimuli. It is well known that all phytohormones synergistically or antagonistically control root growth and development (Cuesta et al. 2013; Jung and McCouch 2013). In Arabidopsis, numerous crucial genes and intricate signaling pathways involved in root development have been well clarified, but many plant species also show their unique molecular and cellular regulatory mechanisms governing root development (Coudert et al. 2010).

Rice (Oryza sativa) is one of the most common crops worldwide. A series of mutants, genes, and genetic mechanisms governing root development have been characterized in rice, some of which are controlled by auxin and 
cytokinin (Mai et al. 2014). For examples, OsAUX1 controls auxin-mediated lateral root initiation through mediating polar auxin transport in rice ( $\mathrm{Yu}$ et al. 2015). Mutation of OsXXT1 causes abnormal root hair development with the reduction of $\mathrm{XyG}$ content and the tensile strength of the cell wall (Wang et al. 2014). Increasing auxin-related mutants including crll (crownless root1), arl1 (adventitious rootless 1), crl4/osgnom1, and oscand1, exhibit abnormal root formation and growth due to the disorders of auxin biosynthesis, polar transport or signaling transduction in rice (Inukai et al. 2005; Liu et al. 2005, 2009; Wang et al. 2011). Moreover,auxin-cytokinin crosstalk plays curial roles in the control of root growth and development in rice. For examples, auxin-induced CRL5 promotes crown root initiation through repressing cytokinin signaling (Kitomi et al. 2011). OsCKX4 and EL5 are essential for crown and lateral root development by mediating crosstalk between auxin and cytokinin pathways (Gao et al. 2014; Koiwai et al. 2007; Mochizuki et al. 2014). In addition, OsMPK $3 / 6$ and OsMKK4/5 are involved in the regulation of root architecture through tuning the interplay of auxin and cytokinin (Singh et al. 2015).

Sugar signals are central in determining plant growth and development by interacting with other signalling pathways (Lastdrager et al. 2014). In Arabidopsis, sucrose affects root architecture through regulating endogenous flavonols accumulation, which can suppress the effect of ABA on root growth (Nguyen et al. 2013). In rice, ABA mediates grain-filling rate by controlling the activities of key enzymes involved in sucrose-to-starch conversion in spikelet (Wang et al. 2015; Zhu et al. 2011). Moreover, several genes involved in sucrose synthesis and metabolism or transport including OSSPS1, OSSUT1, OSSUT2, OSCYT$I N V I$, and $O s c F B P I$ have been proved to be required for the control of rice growth and development (Eom et al. 2011; Hirose et al. 2010, 2014; Jia et al. 2008; Lee et al. 2008). For examples, mutation of OsSUT2 affected sucrose and other sugars transport and caused growth retardation including tiller number, plant height, and root dry weight (Eom et al. 2011). Disruption of OsCYT-INVI impaired normal sucrose metabolism and root growth in rice (Jia et al. 2008).

Recent researches have proved that ethylene and its interaction with ABA play important roles in the control of rice growth and development. For examples, MHZ4/ABA4and MHZ5-mediated ABA signaling is required for ethylene-induced inhibition of root growth (Ma et al. 2014; Yin et al. 2015). Moreover, mhz6/Oseill and mhz7/ein2 exhibit longer root, while overexpression of MHZ6 and MHZ7 represses rice root elongation (Ma et al. 2013; Yang et al. 2015). Importantly, the inducible expression of OSERF2 (Os06g08340) by ethylene in roots was impaired in these mutants (Ma et al. 2013, 2014; Yang et al. 2015; Yin et al. 2015). In this paper, we found that OsERF2 was required for the regulation of primary root growth in rice. The gainof-function mutation of OsERF2 (nsf2857) shows short root, while artificial microRNA-OsERF2 (Ami-OsERF2) lines exhibit longer root, this phenotype is consentient with that of OsEIL1. At the transcript level, OsERF2 negatively regulates expression of candidate genes including $A R F 1$, $M K K 4 / 5, M P K 3 / 6$ and PIN1b/2/9, which are involved in root growth and development through mediating auxin and cytokinin signaling. Furthermore, OsERF2 affects expression of key genes involved in ABA synthesis, which results in the enhanced accumulation of ABA in Ami-OsERF2 lines associated with root growth hypersensitivity to ABA, while those are opposite in nsf2857. Moreover, OsERF2 influence accumulation of sucrose and UDPG through mediating expression of genes related to sucrose metabolism, including INV (Os01g0332100) and OsCYT-INVI, OsSPS2, OsSUS3 and OsSUS6. Particularly, OsCYT-INVI is closely related to the accumulation of sucrose and root growth in rice (Jia et al. 2008). Thus, our results indicate that OsERF2 is required for the control of root architecture and $\mathrm{ABA}$ response by regulating expression of pivotal genes involved in root development, ABA synthesis and sucrose metabolism.

\section{Results}

\section{OsERF2 negative regulates primary root growth}

Recent studies showed that the induction of OsERF2 by ethylene was despaired in several ethylene-insensitive mutants $m h z 4-7$ with longer primary roots (Yang et al. 2015). To investigate whether OsERF2 is involved in the regulation of root growth, we obtained the gain-of-function mutant of OsERF2 (nsf2857) with T-DNA insertion in its promoter from the stock seeds in SHIP (http://ship.plant signal.cn), and generated the artificial microRNA-mediated silencing lines of OsERF2 (Ami-ERF2). After checked expression of OSERF2 in different materials by qPCR (Figure S1), three Ami-OsERF2 lines and nsf2857 were chosen for further analyses. We first compared the root growth status of these materials under normal conditions. For 7-day seedlings, the whole root system of nsf2857 is inferior to that of ZH11. Particularly, the primary root system of $n s f 2857$ (about $6.97 \mathrm{~cm}$ ) was significant shorter than that of ZH11 (about $10.49 \mathrm{~cm}$ ). However, three AmiOSERF2 lines displayed longer primary roots (about $10.20 \mathrm{~cm}$ ) compared with Nip (about $7.04 \mathrm{~cm}$ ) (Fig. 1a, b). These results imply that OsERF2 negatively controls primary root growth, and this phenotype is consistent with that of OsEIL1 (Figure S2). Combined its inducible 


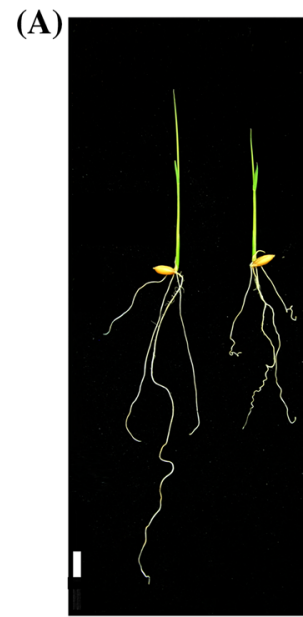

ZH11 nsf2857

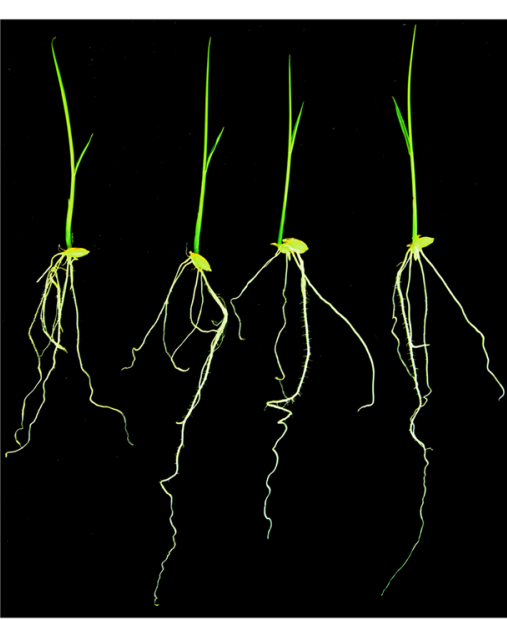

Ami-3
(B)

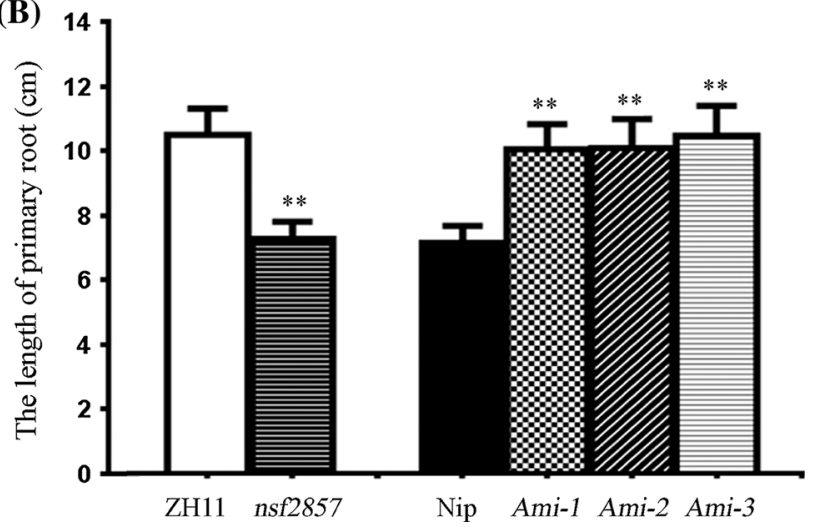

Fig. 1 OsERF2 affects primary root growth. a Comparison of primary root length of 7-day-old seedlings between ZH11 and $n s f 2857$, as well as Nip and three Ami-OsERF2 lines with similar phenotypes, bar $1 \mathrm{~cm}$. b Statistical analyses ( $t$ test) were performed with graphpad prism 6 for the primary root length of ZH11 and $n s f 2857$, as well as Nip and Ami-OsERF2. The seedlings of each sample were more than 20. Error bars represent SD. $* * P<0.01$

expression by key components of ethylene pathway such as OsEIN2 and OsEIL1, we speculate that OsERF2 might act as downstream regulator of ethylene signaling and is required for root growth and development.

\section{OsERF2 regulates expression of key genes related to root architecture}

To analyze the transcriptional regulation of $O s E R F 2$, we compared transcript levels of key genes involved in root growth between in nsf2857, Ami-OsERF2 and WT seedlings. As shown in Fig. 2, expression of 11 candidate genes was downregulated in $n s f 2857$, but upregulated in AmiOsERF2 lines. Among these genes, ARF1, MKK4/5, $M P K 3 / 6$ and PIN1b/2/9 are involved in root growth through mediating auxin or cytokinin signaling. Moreover,
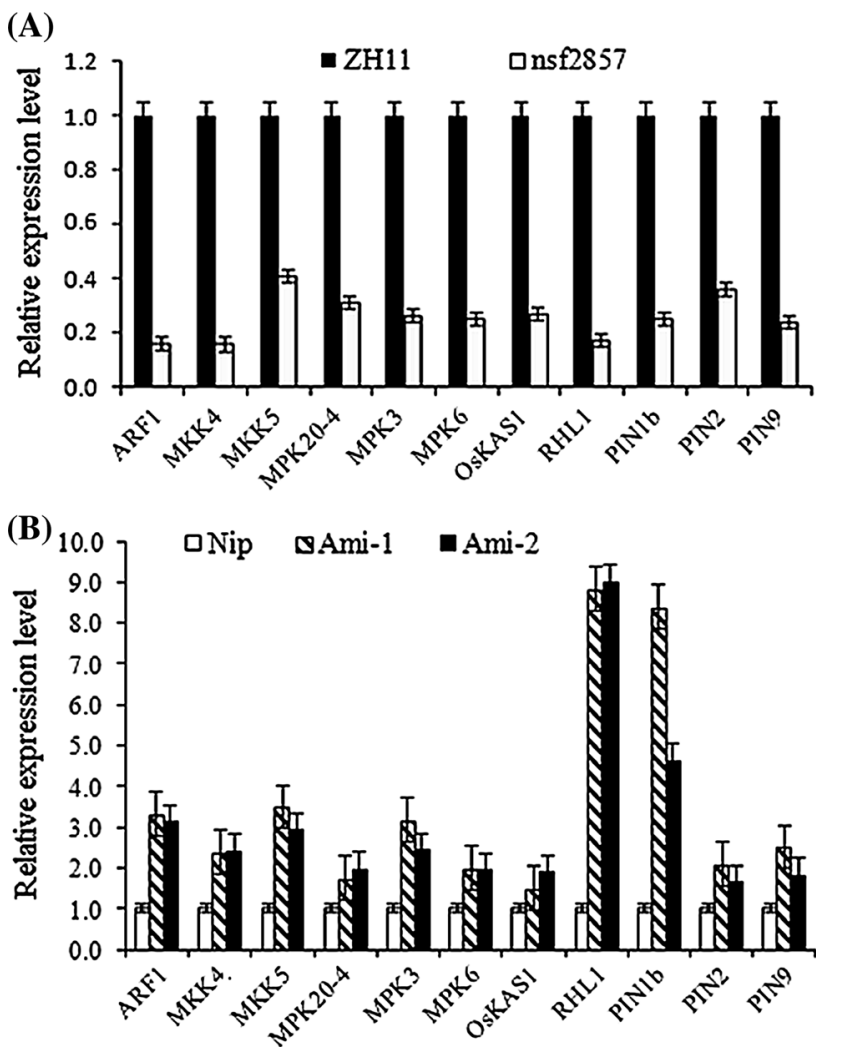

Fig. 2 OsERF2 suppressing expression of key genes involved in root architecture. Relative expression level of candidate genes in $n s f 2857$ roots compared with ZH11 (a) and in Ami-OsERF2 roots compared with Nip (b). Transcript level from ZH11 and Nip was set at 1, respectively. OsACTIN1 was used as an internal control. Data represent the means of three repeats

OsKASI is required for rice root development by regulating fatty acid synthesis (Ding et al. 2015). RHL1, a homologs of Arabidopsis RHL1 (ROOT HAIRLESS 1), is required for root hair initiation (Schneider et al. 1998). These results imply that OsERF2 negatively regulates primary root growth partly due to mediating expression of genes involved in auxin/cytokinin signaling or key functional genes closely related to root development.

\section{OsERF2 affects ABA accumulation and root growth response to $\mathrm{ABA}$ and ethylene}

It has been documented that endogenous ABA positively regulates early root growth in rice (Chen et al. 2006), and the basal levels of endogenous ABA are required for the maintenance of normal root elongation in rice (Ma et al. 2014). In this study, we compared the content of ABA in Ami-OsERF2, nsf 2857 and WT plants. Compared with WT, the content of ABA was decreased in nsf2857, but increased in Ami-OsERF2s (Fig. 3a), suggesting that OsERF2 negatively regulates ABA accumulation. In rice, 
(A)

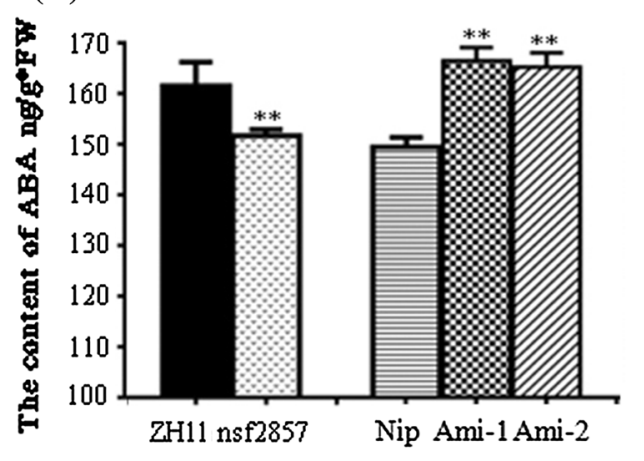

(B)

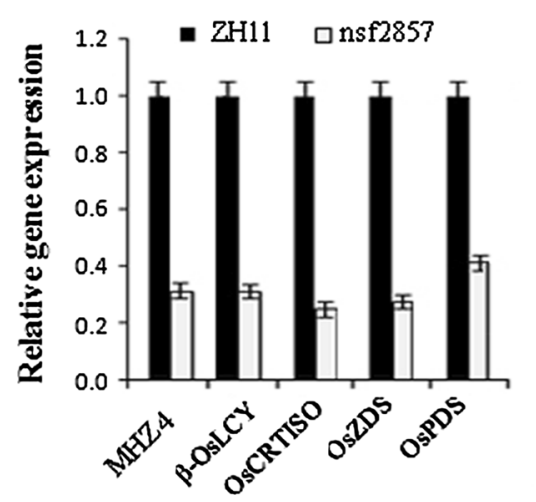

(C)

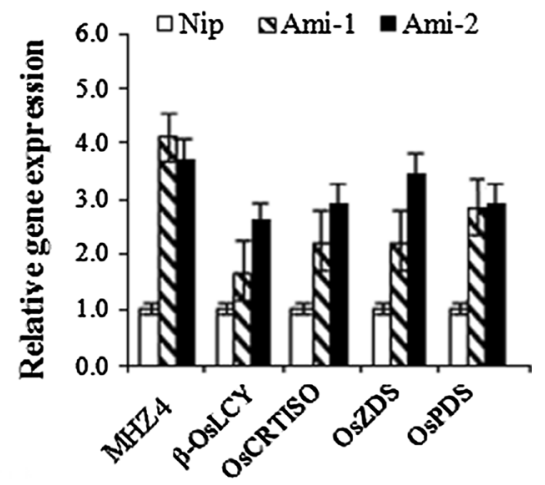

Fig. 3 OsERF2 negatively controls ABA accumulation. a ABA content of 7-day-old seedlings from ZH11, nsf2857, Nip and two AmiOsERF2 lines. Values represent the means SD of three replicates. $* * P<0.01$. b, c Relative expression of ABA biosynthesis genes

MZH4 (ABA4) and pre-harvest sprouting genes ( $P H S \mathrm{~s})$, including OsPDS, OsZDS, OsCRTISO and $\beta$-OsLCY, are essential for the synthesis of ABA (Fang et al. 2008; Ma et al. 2014). Here, we found that expression of these genes was downregulated in $n s f 2857$ (Fig. $3 b$ ), but upregulated in Ami-OsERF2 lines (Fig. 3c). These transcriptional changes were consistent with the accumulation of $\mathrm{ABA}$ in the corresponding materials.

We then analyzed root growth response to $\mathrm{ABA}$ and ethylene treatment in Ami-OsERF2, nsf2857 and WT. When treated with 0.5 and $1 \mu \mathrm{M} \mathrm{ABA}$, the root length of ZH11 decreased from 10.4 to 8.5 and $6.9 \mathrm{~cm}$, respectively, while the root length of nsf 2857 was little changed in the presence of ABA (Fig. 4a, b), indicating that upregulation of OsERF2 caused root insensitivity to ABA in $n s f 2857$. In contrast, down regulation of OsERF2 in Ami-OsERF2 plants resulted in the hypersensitivity of root growth to ABA. As shown in Fig. 3, the average primary root length of three Ami-OsERF2 lines (about $11.41 \mathrm{~cm}$ ) was longer than that of Nip $(8.33 \mathrm{~cm})$ in the absence of ABA. However, the primary root length of Ami-OsERF2 lines decreased to 5.61 and $2.51 \mathrm{~cm}$ under the treatment with 0.5 and $1 \mu \mathrm{M}$ ABA, which were shorter than that of Nip (about 7.63 and $3.78 \mathrm{~cm}$ ) instead, respectively (Fig. 4c, d). The above results suggest that OsERF2 negatively regulates ABA synthesis and root growth response to ABA, which also confirm that the basal level of endogenous $A B A$ is required for the maintenance of normal root elongation in rice.

Upon the ethylene treatment, the primary root growth of ZH11 was significantly inhibited by $64.2 \%$ compared to that in air, but only partially inhibited by $25.7 \%$ in $n s f 2857$. Figure $5 \mathrm{a}, \mathrm{b}$, indicating that $n s f 2857$ showed less ethylene-inhibited root growth. In contrast, the root growth including MHZ4, $\beta$-OsLCY, OsZDS, OsCRTISO and OsPDS in $n s f 2857$ (b), and in two Ami-OsERF2 lines (c). OsACTIN1 was used as an internal control. Data represent the means of three repeats

of Ami-OSERF2 lines was inhibited by more $71 \%$ compared to that in air, and significantly higher than that of Nip $(56 \%)$ (Fig. 5a, b), suggesting that down regulation of $O S E R F 2$ resulted in stronger ethylene-inhibited root growth.

\section{OSERF2 transcriptionally regulates accumulation of sucrose and UDPG}

Sucrose play central roles in the regulation of plant root development by generating a range of signal molecules and interacting with various hormones (Ljung et al. 2015). Here, the contents of sucrose and UDPG (a key precursor for sucrose metabolism) were compared between $n s f 2857$, Ami-OsERF2 and WT plants. Compared with corresponding WT, the sucrose of $n s f 2857$ increased to $112 \%$, while that of Ami-OSERF2 lines decreased to about $84.7 \%$ (Fig. 5a). In contrast to sucrose, the UDPG of $n s f 2857$ $(22.3 \mu \mathrm{g} / \mathrm{mg})$ was $88 \%$ of ZH11 $(25.1 \mu \mathrm{g} / \mathrm{mg})$, while that of Ami-OsERF2 (about $26.2 \mu \mathrm{g} / \mathrm{mg}$ ) was higher than that of NiP $(24.2 \mu \mathrm{g} / \mathrm{mg})$ (Fig. $5 \mathrm{~b})$, suggesting that OsERF2 is involved in regulation of sucrose metabolism. Then, we checked transcripts of several key genes involved in sucrose metabolism including INV (Os01g0332100) and OsCYT-INV1 (encoding neutral invertase), OsSPS2 (encoding sucrose phosphate synthase), OsSUS3 and OsSUS6 (encoding sucrose synthase) between nsf2857, AmiOsERF 2 and WT plants. As shown in Fig. 5c, d, the expression of candidate genes was upregulated in AmiOsERF 2 plants, but downregulated in $n s f 2857$. Particularly, OsCYT-INVI has been reported to be closely related to the accumulation of sucrose and root growth in rice (Jia et al. 2008). Thus, these results suggest that OsERF2 transcriptionally regulates the accumulation of sucrose and 
(A)

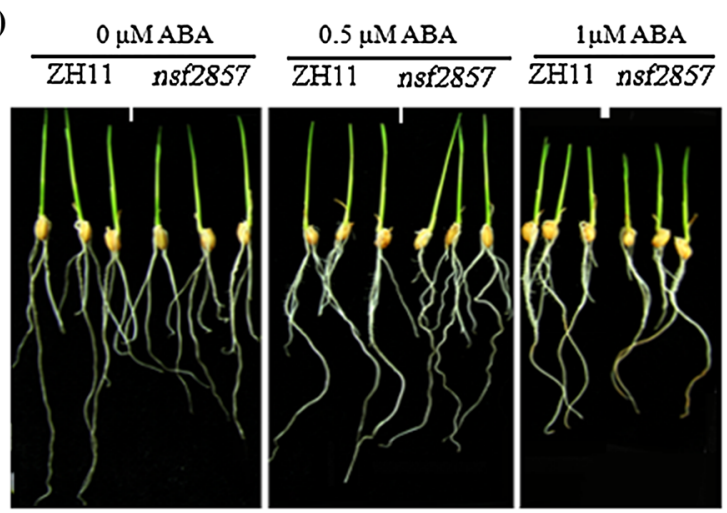

(C)

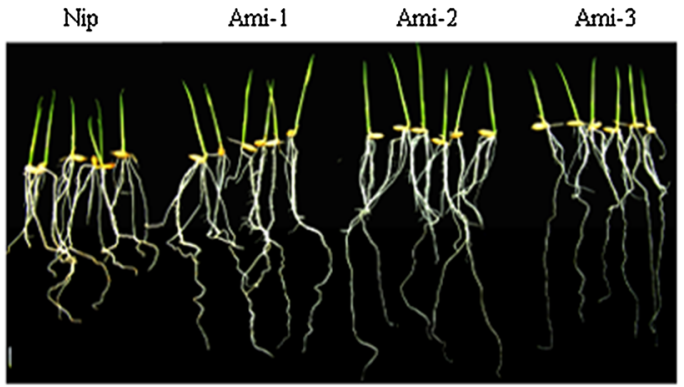

$0.5 \mu \mathrm{M}$ $\mathrm{ABA}$

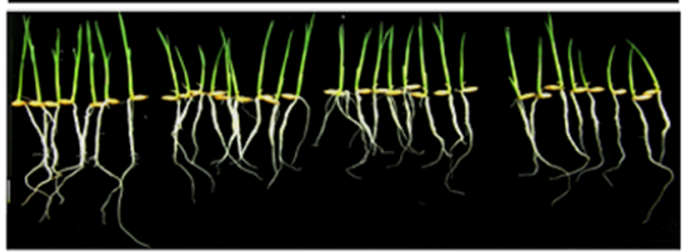

$1 \mu \mathrm{M}$ ABA

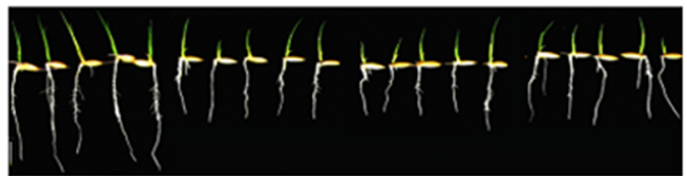

Fig. 4 OsERF2 mediates root growth response to ABA. a 7-Day-old seedlings of $n s f 2857$ exhibit insensitivity to ABA compared with ZH11. c Seedlings of Ami-OsERF2 lines show hypersensitivity to ABA compared with Nip. Bar $1 \mathrm{~cm}$. b, d Statistical analyses $(t$ test) of

UDPG, which partially contribute to its regulatory roles in rice root growth.

\section{Discussion}

The root system is essential for plants to absorb nutrients and water from soils, which determines plant development progress, response to drought and salinity, as well as crop qualities and yield. Rice is very susceptible to water deficit for its shallow root architecture, but the control of root system can increase its yield under drought conditions (Uga et al. 2013). Recently, many genes and mutants involved in root growth and development have been characterized in rice. Some genes are involved in auxin and

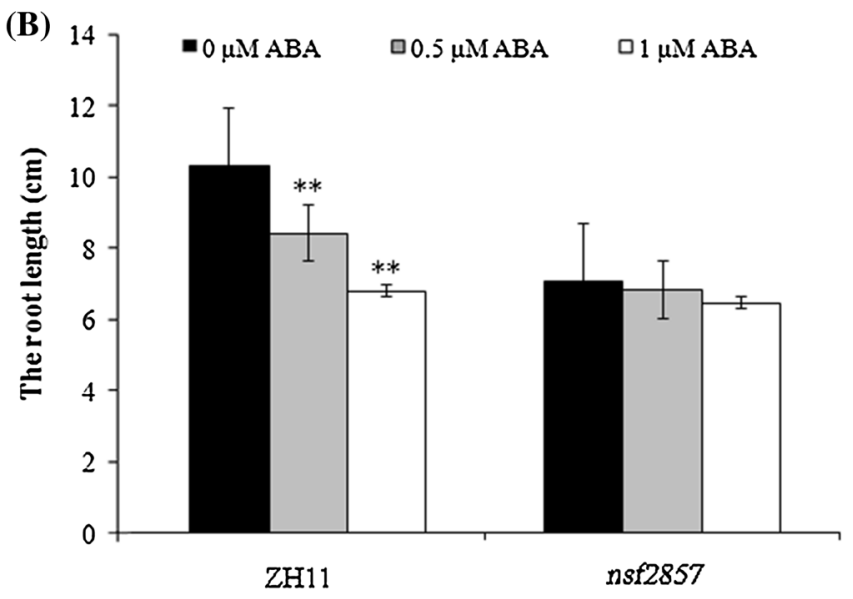

(D)

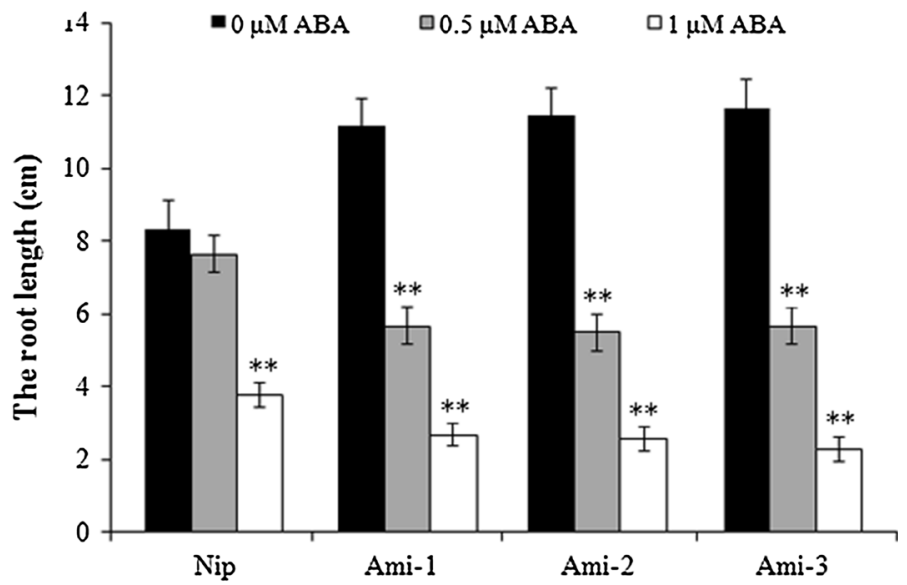

primary root length of 1-week-old seedlings under 0.5 and $1 \mu \mathrm{M}$ ABA were performed with graphpad prism 6. Values represent the means of three independent biological replicates. $* * P<0.01$

cytokinin biosynthesis, transport and homeostasis, and their signal transduction (Azizi et al. 2015; Balzan et al. 2014; Coudert et al. 2010; Mai et al. 2014). For example, the mutation of $C R L 5$, encoding an ERF transcription factor, impairs crown root initiation through repression of cytokinin signaling (Kitomi et al. 2011).

Although auxin and cytokinin govern plant root architecture, the interplay between ethylene and ABA is required for the control of root growth in rice. For examples, etiolated seedlings of Oseill, Osein2, aba4/mhz4 and $m h z 5$ exhibited longer primary roots, while their overexpression resulted in shorter roots, respectively. Moreover, the induction of OsERF 2 by ethylene was disrupted in the roots of these mutants (Ma et al. 2013, 2014; Yang et al. 2015; Yin et al. 2015). In the present study, we evidenced 


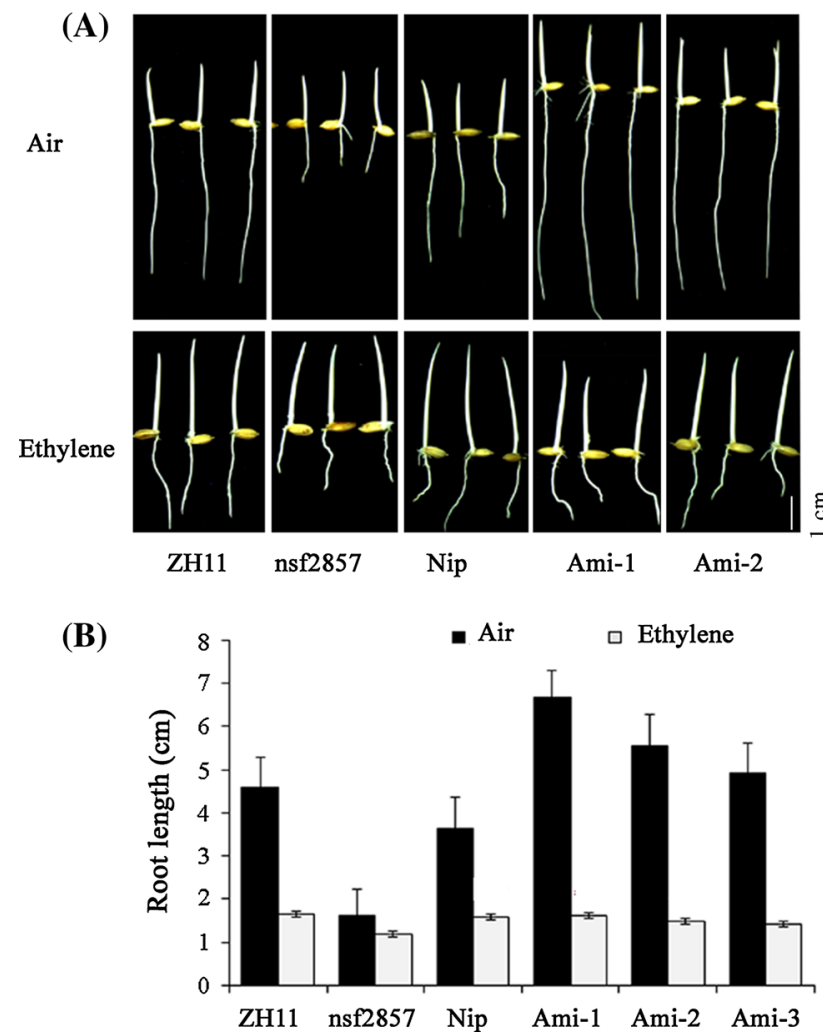

Fig. 5 OsERF2 mediates root growth response to ethylene. a Ethylene response phenotypes in ZH11, nsf2857, Nip and two Ami-OsERF 2 lines. Rice seedlings were grown in dark for 3 days in the presence or absence of $10 \mathrm{ppm}$ ethylene. Bar $1 \mathrm{~cm}$. b Statistical analyses ( $t$ test) of primary root length were performed with graphpad prism 6. Values represent the means of three independent biological replicates. $* * P<0.01$

that the gain-of-function mutant of OsERF2 (nsf2857) exhibited shorter primary root compared to WT, while down regulation of OsERF2 by artificial microRNA (Ami$O S E R F 2)$ resulted in longer primary root, suggesting that $O S E R F 2$ has a similar regulatory effect on the primary root growth with OsEIN2, OsEIL1 and MHZ5. Thus, we conjecture that OsERF2 might act as a downstream component of ethylene pathway and play key roles in the regulation of root growth (Fig. 6).

ERF family factors play multiple roles in the control of plant metabolism, growth and development, and stress response (Licausi et al. 2013). In rice, many ERFs have been reported to be involved in the regulation of response to various stresses by mediating multiple signaling pathways and expression of stress-related genes (Kazan 2015). Importantly, increasing ERF members are involved in the control of development progress in rice. For examples, OsEATB influences plant height, panicle length and branching by regulating gibberellin biosynthesis (Qi et al. 2011). MFS1, SNB and OsIDS1 control inflorescence architecture and floral meristem establishment through tuning expression of spikelet meristem genes (Lee and An 2012; Ren et al. 2013). Moreover, auxin-induced CRL5 promotes crown root initiation via repressing cytokinin signaling (Kitomi et al. 2011). In this study, our findings reveal that OsERF2 negatively regulates expression of pivotal genes related to root growth. Among of these candidate genes, OsMPK3/6, OsMKK4/5 and OsPIN $1 \mathrm{~b} / 9$ are reported to be involved in root growth by tuning auxin and cytokinin signaling (Singh et al. 2015).

ABA affects root growth and development in rice very different from how it does in Arabidopsis, and endogenous ABA positively regulates early root growth and is required for the maintenance of root elongation in rice (Chen et al. 2006; Ma et al. 2014). Here, our results suggest that several genes involved in ABA synthesis, including $M Z H 4$, OsPDS, OsZDS, OsCRTISO and $\beta$-OsLCY, are downregulated in $n s f 2857$ seedlings, but upregulated in Ami-OsERF2 seedlings. These transcription changes are consistent with the increased ABA accumulation in Ami-OsERF2, and the decreased ABA level in nsf2857. Importantly, it has been proved that MHZ4 is responsible for ABA biosynthesis and involved in the control of ethylene response, whose mutation and overexpression causes reduced and enhanced ethylene-inhibited root growth, respectively (Ma et al. 2014). In this paper, we found that Ami-OSERF2 lines exhibited longer primary roots and enhanced sensitivity to ABA- and ethylene-treatment, while these phenotypes were the opposite in $n s f 2857$. Moreover, our results showed that upregulation of $O s E R F 2$ caused the decreased expression of MHZ4 and ABA accumulation in nsf 2857 , which displayed similar phenotypes with mhz4 including the shorter roots and reduced ABA- and ethylene-responses. However, Ami-OsERF2 plants exhibited similar phenotypes with transgenic rice overexpressing MHZ4. Taken together, we speculate that OsERF2 is required for the interplay between ethylene and $\mathrm{ABA}$ in control of rice root growth, which might be partially related with its transcriptional control of $M H Z 4$ expression and ABA accumulation.

As the main assimilated carbon of photosynthesis, sucrose play central roles in plant growth and development, as well as stress response by generating series of sugars as metabolites or as signaling molecules interacting the cross talks of metabolic, hormonal, and stress signals (Ljung et al. 2015; Ruan 2014). In Arabidopsis, sucrose inhibits hypocotyl elongation and promotes root growth by tuning auxin signal and cotyledon-derived long-distance signal (Kircher and Schopfer 2012; Stokes et al. 2013). In rice, mutation of OSSPS1, OsSUT1, OsSUT2, and OscFBP1 causes growth retardation and abnormal pollen development by affecting sucrose synthesis or transporter (Eom et al. 2011; Hirose et al. 2010, 2014; Lee et al. 2008). Similarly, UDP-glucose (UDPG) is a key metabolite for the 

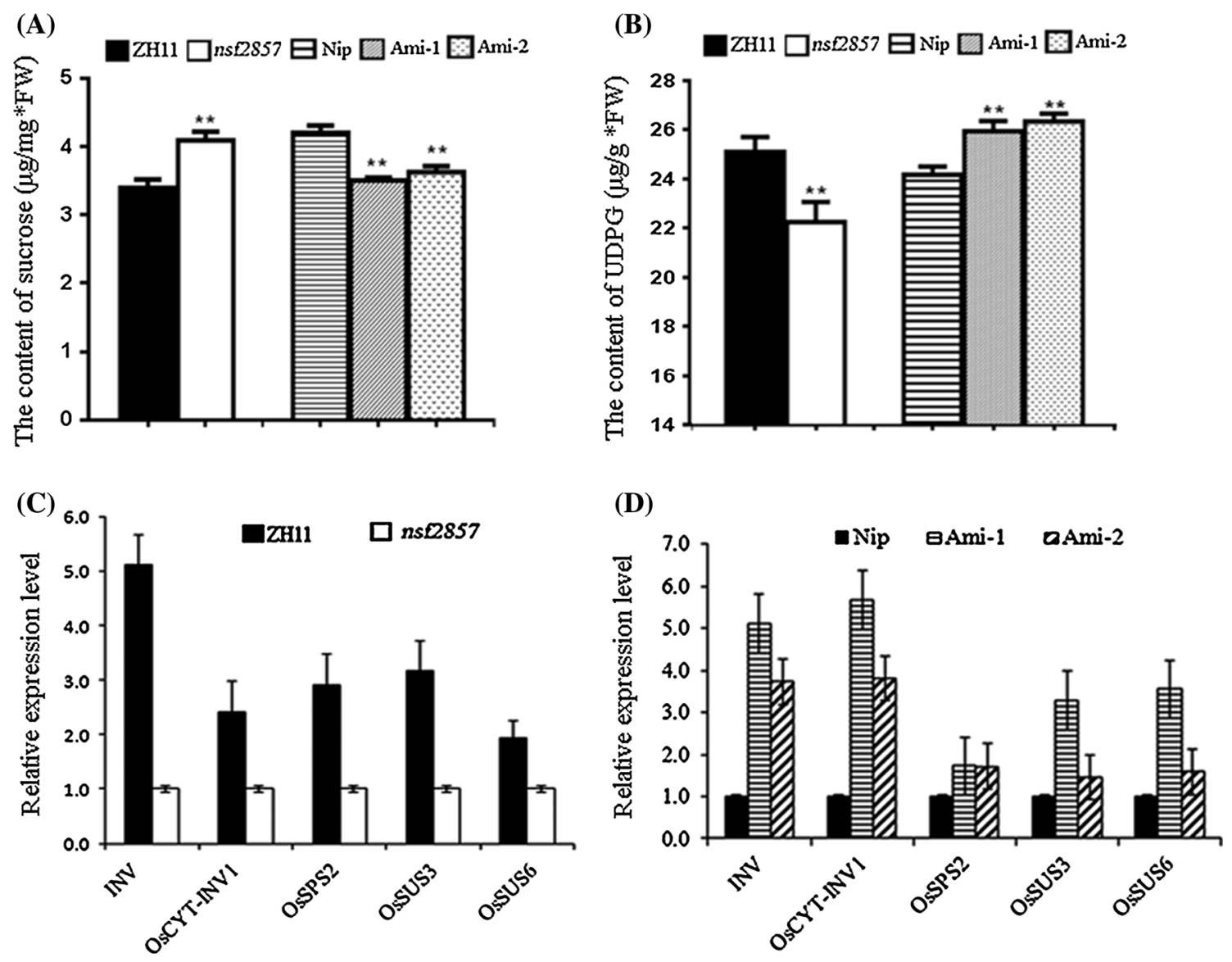

Fig. 6 OsERF2 transcriptionally influences the accumulation of sucrose and UDPG. a Sucrose content was increased in $n s f 2857$, but decreased in Ami-OsERF 2 lines. b UDPG content was decreased in $n s f 2857$, but increased in Ami-OsERF2 lines. Statistical analyses ( $t$ test) of sucrose and UDPG contents were performed with graphpad

prism 6. Values represent the means of three replicates. $* * P<0.01$. c, $\mathbf{d}$ Relative expression of key genes involved in sucrose metabolism in $n s f 2857$ and Ami-OsERF 2 lines. OsACTIN1 was used as an internal control. Data represent the means of three repeats

synthesis of sucrose, polysaccharides, glycoproteins, glycolipids and myriads of glycosylated secondary metabolites (Decker et al. 2012). UGPase plays important roles in carbohydrate metabolism through catalyzing the reversible conversion of glucose-1-phosphate to UDPG, which is essential for plant growth, pollen development, and male sterility in rice and Arabidopsis (Chen et al. 2007; Park et al. 2010; Woo et al. 2008). In rice, expression levels of genes involved in sucrose metabolism are correlated with sucrose accumulation (Maruyama et al. 2014). In this paper, we found that upregulation of OsERF2 resulted in the decreased sucrose and the increased UDPG in $n s f 2857$ plants, while it was the opposite in Ami-OsERF2 plants. Consistent with these physiological changes, expression of key genes closely related to sucrose metabolism including $I N V$ and OsCYT-INV1, OsSPS2, OsSUS3, and OsSUS6, were downregulated in $n s f 2857$, but upregulated in $A m i$ OSERF2 lines. Particularly, mutation of OsCYT-INVI has been proved to cause the enhanced sucrose level and the reduced root growth in rice (Jia et al. 2008). Consistent

with this result, we found that OsCYT-INVI was downregulated in $n s f 2857$, which exhibited short primary root and increased accumulation of sucrose. Thus, these findings suggest that OsERF2 transcriptionally controls the accumulation of sucrose and UDPG, which might further trigger the complex alteration of carbohydrate metabolism and multiple signaling pathways, and partially account for the effect of OsERF2 on rice root growth.

\section{Materials and methods}

\section{Rice materials and treatments}

In this study, rice (O. sativa L. subsp. japonica cv Zhonghua 11 (ZH11) and $O$. sativa L. subsp. japonica cv nipponbare (Nip) were used as the wild-type (WT) for $n s f 2857$ and amiERF2, respectively. For root growth and ABA response, more 40 geminated seeds from all samples were planted in 1/2 Murashige and Skoog (MS) medium solution 
supplemented with $0,0.5$ and $1 \mu \mathrm{M}$ ABA. After growth for 7 days, root lengths were measured and photographed. For measurement of ABA, sucrose and UDPG, geminated seeds from all samples were planted in 1/2 Murashige and Skoog (MS) medium solution for 2 weeks. All data are presented as means of three times. For ethylene treatment, rice seedlings were grown on a stainless steel sieve placed in an air-tight plastic box with $10 \mathrm{ppm}$ ethylene and in air as control. The seedlings were incubated at $28{ }^{\circ} \mathrm{C}$ in the dark for 3 days.

Artificial microRNA (amiRNA-OsERF2) construct was generated using specific primer sets (Table S1) designed through programs available on the (http://wmd3.weigel world.org/), and cloned into the binary vectors pCAMBIA5300. amiRNA-OsERF2 plants were generated by Agrobacterium-mediated transformation and examined by qRT-PCR with specific primers (Table S1). The seeds of oseill mutant and OsEIL1-OX were obtained from Dr. Jinsong Zhang.

\section{Measurements of ABA, sucrose and UDPG}

ABA extraction was according to Zhu et al. (2010) measured using the ELISA kit (CSB-E09159Pl, China Agricultural University, China). $0.2 \mathrm{~g}$ leaves of 2-week-old seedlings were homogenized and extracted overnight at $4{ }^{\circ} \mathrm{C}$ in $80 \%$ methanol by vortex spinning. After centrifuged and concentrated to approximately $50 \mu \mathrm{l}$, these extracts were suspended with $1 \mathrm{ml}$ TBS buffer $(25 \mathrm{mM}$ Tris- $\mathrm{HCl} \mathrm{pH} \mathrm{7.5,} 100 \mathrm{mM} \mathrm{NaCl}, 1 \mathrm{mM} \mathrm{MgCl} 2$, and $3 \mathrm{mM}$ NaN3) and used for ELISA according to the manufacturer's instructions. ABA concentrations were calculated as $\mathrm{ng} / \mathrm{g}$ fresh weight. Each measurement was replicated three times.

Sucrose and UDPG content was measured by HPLC according to Bahaji et al. (2015). $0.1 \mathrm{~g} 2$-week-old seedlings were ground in liquid nitrogen. For measurement of sucrose, the powder was suspended in $1 \mathrm{ml}$ of $90 \%$ ethanol, left at $70{ }^{\circ} \mathrm{C}$ for $90 \mathrm{~min}$ and centrifuged at $13,000 \times \mathrm{g}$ for $10 \mathrm{~min}$. For measurement of UDPG, the powder was suspended in $1 \mathrm{ml}$ of $1 \mathrm{M} \mathrm{HClO}_{4}$, left at $4{ }^{\circ} \mathrm{C}$ for $2 \mathrm{~h}$ and centrifuged at $13,000 \times g$ for $10 \mathrm{~min}$. The supernatant was neutralized with $\mathrm{K}_{2} \mathrm{CO}_{3}$ and centrifuged at $13,000 \times g$ for $10 \mathrm{~min}$. Sucrose was determined by HPLC with UPLC WATERS Acquity system by gradient separation with $\mathrm{BEH}$ Amide $2.1 \times 100 \mathrm{~mm} 1.7 \mu \mathrm{m}$. UDPG was measured by HPLC on a system obtained from Waters Associates fitted with ZORBAX Carbohydrate $4.6 \times 250 \mathrm{~mm} 5 \mu \mathrm{m}$. Standard sucrose and UDPG were purchased from Sigma.

\section{Gene expression analysis}

After geminated seeds were planted in 1/2 MS medium solution for 7 days, total RNA of roots or leaves was isolated from all samples using TRIzol reagent (Invitrogen, Beijing, China) and used to synthesize first-strand cDNA with All-in-One ${ }^{\mathrm{TM}}$ First-Strand cDNA Synthesis Kit (Transgen, China). RT-qPCR was performed using genespecific primers (Table S1) with TransStart Green qPCR SuperMix (Transgen, China). OsActinl was used as the internal control. All experiments were repeated three times, and the average was calculated.

Acknowledgments We are grateful to Dr. Jin-Song Zhang from Institute of Genetics and Developmental Biology (Chinese Academy of Sciences) for his kind sharing oseil1 mutant and OsEIL1-OX seeds. This work was supported by the Major Special Foundation of Transgenic Plants in China (2013ZX08001003 and 2014ZX0800915B), and by National Natural Science Foundation of China (Grant No. 31172025).

Open Access This article is distributed under the terms of the Creative Commons Attribution 4.0 International License (http://crea tivecommons.org/licenses/by/4.0/), which permits unrestricted use, distribution, and reproduction in any medium, provided you give appropriate credit to the original author(s) and the source, provide a link to the Creative Commons license, and indicate if changes were made.

\section{References}

Azizi P, Rafii MY, Maziah M, Abdullah SN, Hanafi MM, Latif MA, Rashid AA, Sahebi M (2015) Understanding the shoot apical meristem regulation: a study of the phytohormones, auxin and cytokinin, in rice. Mech Dev 135:1-15

Bahaji A, Baroja-Fernandez E, Ricarte-Bermejo A, Sanchez-Lopez AM, Munoz FJ, Romero JM, Ruiz MT, Baslam M, Almagro G, Sesma MT, Pozueta-Romero J (2015) Characterization of multiple SPS knockout mutants reveals redundant functions of the four Arabidopsis sucrose phosphate synthase isoforms in plant viability, and strongly indicates that enhanced respiration and accelerated starch turnover can alleviate the blockage of sucrose biosynthesis. Plant Sci 238:135-147

Balzan S, Johal GS, Carraro N (2014) The role of auxin transporters in monocots development. Front Plant Sci 5:393

Chen CW, Yang YW, Lur HS, Tsai YG, Chang MC (2006) A novel function of abscisic acid in the regulation of rice (Oryza sativa L.) root growth and development. Plant Cell Physiol 47:1-13

Chen R, Zhao X, Shao Z, Wei Z, Wang Y, Zhu L, Zhao J, Sun M, He $\mathrm{R}$, He G (2007) Rice UDP-glucose pyrophosphorylase1 is essential for pollen callose deposition and its cosuppression results in a new type of thermosensitive genic male sterility. Plant Cell 19:847-861

Coudert Y, Perin C, Courtois B, Khong NG, Gantet P (2010) Genetic control of root development in rice, the model cereal. Trends Plant Sci 15:219-226 
Cuesta C, Wabnik K, Benkova E (2013) Systems approaches to study root architecture dynamics. Front Plant Sci 4:537

Decker D, Meng M, Gornicka A, Hofer A, Wilczynska M, Kleczkowski LA (2012) Substrate kinetics and substrate effects on the quaternary structure of barley UDP-glucose pyrophosphorylase. Phytochemistry 79:39-45

Ding W, Lin L, Zhang B, Xiang X, Wu J, Pan Z, Zhu S (2015) OsKASI, a $\beta$-ketoacyl-[acyl carrier protein] synthase $I$, is involved in root development in rice (Oryza sativa L.). Planta 242:203-213

Eom JS, Cho JI, Reinders A, Lee SW, Yoo Y, Tuan PQ, Choi SB, Bang G, Park YI, Cho MH, Bhoo SH, An G, Hahn TR, Ward JM, Jeon JS (2011) Impaired function of the tonoplast-localized sucrose transporter in rice, OsSUT2, limits the transport of vacuolar reserve sucrose and affects plant growth. Plant Physiol 157:109-119

Fang J, Chai C, Qian Q, Li C, Tang J, Sun L, Huang Z, Guo X, Sun C, Liu M, Zhang Y, Lu Q, Wang Y, Lu C, Han B, Chen F, Cheng Z, Chu C (2008) Mutations of genes in synthesis of the carotenoid precursors of ABA lead to pre-harvest sprouting and photooxidation in rice. Plant $\mathrm{J} 54: 177-189$

Gao S, Fang J, Xu F, Wang W, Sun X, Chu J, Cai B, Feng Y, Chu C (2014) CYTOKININ OXIDASE/DEHYDROGENASE4 integrates cytokinin and auxin signaling to control rice crown root formation. Plant Physiol 165:1035-1046

Hirose T, Zhang Z, Miyao A, Hirochika H, Ohsugi R, Terao T (2010) Disruption of a gene for rice sucrose transporter, OsSUT1, impairs pollen function but pollen maturation is unaffected. J Exp Bot 61:3639-3646

Hirose T, Hashida Y, Aoki N, Okamura M, Yonekura M, Ohto C, Terao T, Ohsugi R (2014) Analysis of gene-disruption mutants of a sucrose phosphate synthase gene in rice, OsSPS1, shows the importance of sucrose synthesis in pollen germination. Plant Sci 225:102-106

Inukai Y, Sakamoto T, Ueguchi-Tanaka M, Shibata Y, Gomi K, Umemura I, Hasegawa Y, Ashikari M, Kitano H, Matsuoka M (2005) Crown rootless1, which is essential for crown root formation in rice, is a target of an AUXIN RESPONSE FACTOR in auxin signaling. Plant Cell 17:1387-1396

Jia L, Zhang B, Mao C, Li J, Wu Y, Wu P, Wu Z (2008) OsCYTINV1 for alkaline/neutral invertase is involved in root cell development and reproductivity in rice (Oryza sativa L.). Planta 228:51-59

Jung JK, McCouch S (2013) Getting to the roots of it: genetic and hormonal control of root architecture. Front Plant Sci 4:186

Kazan K (2015) Diverse roles of jasmonates and ethylene in abiotic stress tolerance. Trends Plant Sci 20:219-229

Kircher S, Schopfer P (2012) Photosynthetic sucrose acts as cotyledon-derived long-distance signal to control root growth during early seedling development in Arabidopsis. Proc Natl Acad Sci USA 109:11217-11221

Kitomi Y, Ito H, Hobo T, Aya K, Kitano H, Inukai Y (2011) The auxin responsive AP2/ERF transcription factor CROWN ROOTLESS5 is involved in crown root initiation in rice through the induction of OsRR1, a type-A response regulator of cytokinin signaling. Plant J 67:472-484

Koiwai H, Tagiri A, Katoh S, Katoh E, Ichikawa H, Minami E, Nishizawa Y (2007) RING-H2 type ubiquitin ligase EL5 is involved in root development through the maintenance of cell viability in rice. Plant J 51:92-104

Lastdrager J, Hanson J, Smeekens S (2014) Sugar signals and the control of plant growth and development. J Exp Bot 65:799-807

Lee DY, An G (2012) Two AP2 family genes, supernumerary bract (SNB) and Osindeterminate spikelet 1 (OsIDS1), synergistically control inflorescence architecture and floral meristem establishment in rice. Plant J 69:445-461
Lee SK, Jeon JS, Bornke F, Voll L, Cho JI, Goh CH, Jeong SW, Park YI, Kim SJ, Choi SB, Miyao A, Hirochika H, An G, Cho MH, Bhoo SH, Sonnewald U, Hahn TR (2008) Loss of cytosolic fructose-1,6-bisphosphatase limits photosynthetic sucrose synthesis and causes severe growth retardations in rice (Oryza sativa). Plant, Cell Environ 31:1851-1863

Licausi F, Ohme-Takagi M, Perata P (2013) APETALA2/Ethylene Responsive Factor (AP2/ERF) transcription factors: mediators of stress responses and developmental programs. New Phytol 199:639-649

Liu H, Wang S, Yu X, Yu J, He X, Zhang S, Shou H, Wu P (2005) ARL1, a LOB-domain protein required for adventitious root formation in rice. Plant $\mathrm{J}$ 43:47-56

Liu S, Wang J, Wang L, Wang X, Xue Y, Wu P, Shou H (2009) Adventitious root formation in rice requires OsGNOM1 and is mediated by the OsPINs family. Cell Res 19:1110-1119

Ljung K, Nemhauser JL, Perata P (2015) New mechanistic links between sugar and hormone signalling networks. Curr Opin Plant Biol 25:130-137

Ma B, He SJ, Duan KX, Yin CC, Chen H, Yang C, Xiong Q, Song QX, Lu X, Chen HW, Zhang WK, Lu TG, Chen SY, Zhang JS (2013) Identification of rice ethylene-response mutants and characterization of MHZ7/OsEIN2 in distinct ethylene response and yield trait regulation. Mol Plant 6:1830-1848

Ma B, Yin CC, He SJ, Lu X, Zhang WK, Lu TG, Chen SY, Zhang JS (2014) Ethylene-induced inhibition of root growth requires abscisic acid function in rice (Oryza sativa L.) seedlings. PLoS Genet 10:e1004701

Mai CD, Phung NT, To HT, Gonin M, Hoang GT, Nguyen KL, Do VN, Courtois B, Gantet P (2014) Genes controlling root development in rice. Rice (N Y) 7:30

Maruyama K, Urano K, Yoshiwara K, Morishita Y, Sakurai N, Suzuki H, Kojima M, Sakakibara H, Shibata D, Saito K, Shinozaki K, Yamaguchi-Shinozaki K (2014) Integrated analysis of the effects of cold and dehydration on rice metabolites, phytohormones, and gene transcripts. Plant Physiol 164:1759-1771

Mochizuki S, Jikumaru Y, Nakamura H, Koiwai H, Sasaki K, Kamiya Y, Ichikawa H, Minami E, Nishizawa Y (2014) Ubiquitin ligase EL5 maintains the viability of root meristems by influencing cytokinin-mediated nitrogen effects in rice. J Exp Bot 65:2307-2318

Nguyen HN, Kim JH, Hyun WY, Nguyen NT, Hong SW, Lee H (2013) TTG1-mediated flavonols biosynthesis alleviates root growth inhibition in response to ABA. Plant Cell Rep 32:503-514

Park JI, Ishimizu T, Suwabe K, Sudo K, Masuko H, Hakozaki H, Nou IS, Suzuki G, Watanabe M (2010) UDP-glucose pyrophosphorylase is rate limiting in vegetative and reproductive phases in Arabidopsis thaliana. Plant Cell Physiol 51:981-996

Qi W, Sun F, Wang Q, Chen M, Huang Y, Feng YQ, Luo X, Yang J (2011) Rice ethylene-response AP2/ERF factor OsEATB restricts internode elongation by down-regulating a gibberellin biosynthetic gene. Plant Physiol 157:216-228

Ren D, Li Y, Zhao F, Sang X, Shi J, Wang N, Guo S, Ling Y, Zhang C, Yang Z, He G (2013) MULTI-FLORET SPIKELET1, which encodes an AP2/ERF protein, determines spikelet meristem fate and sterile lemma identity in rice. Plant Physiol 162:872-884

Ruan YL (2014) Sucrose metabolism: gateway to diverse carbon use and sugar signaling. Annu Rev Plant Biol 65:33-67

Schneider K, Mathur J, Boudonck K, Wells B, Dolan L, Roberts K (1998) The ROOT HAIRLESS 1 gene encodes a nuclear protein required for root hair initiation in Arabidopsis. Genes Dev 12:2013-2021

Singh P, Mohanta TK, Sinha AK (2015) Unraveling the intricate nexus of molecular mechanisms governing rice root development: OsMPK3/6 and auxin-cytokinin interplay. PLoS ONE 10:e0123620 
Stokes ME, Chattopadhyay A, Wilkins O, Nambara E, Campbell MM (2013) Interplay between sucrose and folate modulates auxin signaling in Arabidopsis. Plant Physiol 162:1552-1565

Uga Y, Sugimoto K, Ogawa S, Rane J, Ishitani M, Hara N, Kitomi Y, Inukai Y, Ono K, Kanno N, Inoue H, Takehisa H, Motoyama R, Nagamura Y, Wu J, Matsumoto T, Takai T, Okuno K, Yano M (2013) Control of root system architecture by DEEPER ROOTING 1 increases rice yield under drought conditions. Nat Genet 45:1097-1102

Wang XF, He FF, Ma XX, Mao CZ, Hodgman C, Lu CG, Wu P (2011) OsCAND1 is required for crown root emergence in rice. Mol Plant 4:289-299

Wang C, Li S, Ng S, Zhang B, Zhou Y, Whelan J, Wu P, Shou H (2014) Mutation in xyloglucan 6-xylosytransferase results in abnormal root hair development in Oryza sativa. J Exp Bot 65:4149-4157

Wang Z, Xu Y, Chen T, Zhang H, Yang J, Zhang J (2015) Abscisic acid and the key enzymes and genes in sucrose-to-starch conversion in rice spikelets in response to soil drying during grain filling. Planta 241:1091-1107

Woo MO, Ham TH, Ji HS, Choi MS, Jiang W, Chu SH, Piao R, Chin JH, Kim JA, Park BS, Seo HS, Jwa NS, McCouch S, Koh HJ (2008) Inactivation of the UGPase1 gene causes genic male sterility and endosperm chalkiness in rice (Oryza sativa L.). Plant J 54:190-204
Yang C, Ma B, He SJ, Xiong Q, Duan KX, Yin CC, Chen H, Lu X, Chen SY, Zhang JS (2015) MHZ6/OsEIL1 and OsEIL2 regulate ethylene response of roots and coleoptiles and negatively affect salt tolerance in rice. Plant Physiol. doi:10.1104/pp.15.00353

Yin CC, Ma B, Collinge DP, Pogson BJ, He SJ, Xiong Q, Duan KX, Chen H, Yang C, Lu X, Wang YQ, Zhang WK, Chu CC, Sun XH, Fang S, Chu JF, Lu TG, Chen SY, Zhang JS (2015) Ethylene responses in rice roots and coleoptiles are differentially regulated by a carotenoid isomerase-mediated abscisic acid pathway. Plant Cell 27:1061-1081

Yu C, Sun C, Shen C, Wang S, Liu F, Liu Y, Chen Y, Li C, Qian Q, Aryal B, Geisler M, de Jiang A, Qi Y (2015) The auxin transporter, OsAUX1, is involved in primary root and root hair elongation and in Cd stress responses in rice (Oryza sativa L.). Plant J 83:818-830

Zhu Y, Dun X, Zhou Z, Xia S, Yi B, Wen J, Shen J, Ma C, Tu J, Fu T (2010) A separation defect of tapetum cells and microspore mother cells results in male sterility in Brassica napus: the role of abscisic acid in early anther development. Plant Mol Biol 72:111-123

Zhu G, Ye N, Yang J, Peng X, Zhang J (2011) Regulation of expression of starch synthesis genes by ethylene and ABA in relation to the development of rice inferior and superior spikelets. J Exp Bot 62:3907-3916 